University of Texas Rio Grande Valley

ScholarWorks @ UTRGV

$9-15-2012$

\title{
COP1 Mediates the Coordination of Root and Shoot Growth by Light Through Modulation of PIN1- and Pin2-Dependent Auxin Transport in ArabidoPsis
}

\author{
Massimiliano Sassi \\ Yanfen Lu \\ Yonghong Zhang \\ The University of Texas Rio Grande Valley, yonghong.zhang@utrgv.edu \\ Juan Wang \\ Pankaj Dhonukshe
}

See next page for additional authors

Follow this and additional works at: https://scholarworks.utrgv.edu/chem_fac

Part of the Chemistry Commons

\section{Recommended Citation}

Mao, Y. (2012). Facile molten-salt synthesis of double perovskite La2BMnO6 nanoparticles. RSC Advances, 2(33), 12675-12678. https://doi.org/10.1039/C2RA21789A

This Article is brought to you for free and open access by the College of Sciences at ScholarWorks @ UTRGV. It has been accepted for inclusion in Chemistry Faculty Publications and Presentations by an authorized administrator of ScholarWorks@ UTRGV. For more information, please contact justin.white@utrgv.edu,william.flores01@utrgv.edu. 


\section{Authors}

Massimiliano Sassi, Yanfen Lu, Yonghong Zhang, Juan Wang, Pankaj Dhonukshe, Ikram Blilou, Minqiu Dai, Juan Li, Ximing Gong, Yvon Jaillais, Xuhong Yu, Jan Traas, Ida Ruberti, Haiyang Wang, Ben Scheres, Teva VernouX, and Jian $\mathrm{Xu}$ 


\title{
COP1 mediates the coordination of root and shoot growth by light through modulation of PIN1- and PIN2-dependent auxin transport in Arabidopsis
}

\author{
Massimiliano Sassi ${ }^{1,2}$, Yanfen Lu $^{3, *}$, Yonghong Zhang $4, *$, Juan Wang ${ }^{3, *}$, Pankaj Dhonukshe ${ }^{5}$, Ikram Blilou ${ }^{5}$, \\ Minqiu Dai ${ }^{6}$, Juan $\mathrm{Li}^{4}$, Ximing Gong ${ }^{3}$, Yvon Jaillais ${ }^{1}$, Xuhong Yu ${ }^{7}$, Jan Traas ${ }^{1}$, Ida Ruberti ${ }^{2}$, Haiyang Wang ${ }^{6}$, \\ Ben Scheres ${ }^{5}$, Teva Vernoux ${ }^{1, \pm}$ and Jian $\mathrm{Xu}^{3,4,5, \pm}$
}

\begin{abstract}
SUMMARY
When a plant germinates in the soil, elongation of stem-like organs is enhanced whereas leaf and root growth is inhibited. How these differential growth responses are orchestrated by light and integrated at the organismal level to shape the plant remains to be elucidated. Here, we show that light signals through the master photomorphogenesis repressor COP1 to coordinate root and shoot growth in Arabidopsis. In the shoot, COP1 regulates shoot-to-root auxin transport by controlling the transcription of the auxin efflux carrier gene PIN-FORMED1 (PIN1), thus appropriately tuning shoot-derived auxin levels in the root. This in turn directly influences root elongation and adapts auxin transport and cell proliferation in the root apical meristem by modulating PIN1 and PIN2 intracellular distribution in the root in a COP1-dependent fashion, thus permitting a rapid and precise tuning of root growth to the light environment. Our data identify auxin as a long-distance signal in developmental adaptation to light and illustrate how spatially separated control mechanisms can converge on the same signaling system to coordinate development at the whole plant level.
\end{abstract}

KEY WORDS: Arabidopsis, Root growth, Light, Auxin transport, COP1, PIN1, PIN2

\section{INTRODUCTION}

A striking example of the plasticity of plant development (Casal et al., 2004; Matesanz et al., 2010; Nicotra et al., 2010) is revealed when a seedling emerges through the soil. In the absence of light, the hypocotyl elongates whereas cotyledons remain closed. When the seedling emerges from the soil, hypocotyl elongation stops and the cotyledons open, exposing the young leaves to light (Nemhauser and Chory, 2002; Quail, 2002). Two distinct developmental programs, known as photomorphogenesis and skotomorphogenesis (or etiolation) in light and in darkness, respectively, control these radically different growth patterns (Nemhauser and Chory, 2002; Quail, 2002).

Genetic analysis of the CONSTITUTIVE PHOTOMORPHOGENIC/DE-ETIOLATED/FUSCA (COP/DET/ FUS) loci of Arabidopsis thaliana (hereafter Arabidopsis) led to substantial insights into the molecular mechanisms regulating lightmediated morphogenesis (Yi and Deng, 2005; Li et al., 2011). The cop/det/fus mutants display a de-etiolated phenotype when grown in darkness and most of them are defective in the components of a macromolecular complex named the COP9 signalosome (CSN).

\footnotetext{
${ }^{1}$ CNRS, INRA, ENS Lyon, UCBL, Université de Lyon, Laboratoire de Reproduction et Développement des Plantes, 46 Allée d'Italie, 69364 Lyon Cedex 07, France. ${ }^{2}$ Istituto di Biologia e Patologia Molecolari, CNR, Piazzale Aldo Moro 5, 00185 Rome, Italy. ${ }^{3}$ Department of Biological Sciences and NUS Centre for Biolmaging Sciences, National University of Singapore, 14 Science Drive 4, Singapore 117543. ${ }^{4}$ National Key Laboratory of Crop Genetic Improvement, Huazhong Agricultural University, Wuhan 430070, China. ${ }^{5}$ Department of Molecular Genetics, Utrecht University, Padualaan 8, 3584CH Utrecht, The Netherlands. ${ }^{6}$ Department of Molecular, Cellular, and Developmental Biology, Yale University, New Haven, CT 06520-8104, USA. ${ }^{7}$ Department of Biology, Indiana University, Bloomington, IN 47405, USA

* These authors contributed equally to this work

‡Authors for correspondence (teva.vernoux@ens-lyon.fr; dbsxj@nus.edu.sg)
}

The CSN regulates the $26 \mathrm{~S}$ proteasome-mediated degradation of photomorphogenesis-promoting transcription factors, a central step in the regulation of developmental responses to light (Yi and Deng, 2005; Li et al., 2011). The RING E3 ubiquitin ligase CONSTITUTIVE PHOTOMORPHOGENIC1 (COP1) ubiquitinates these transcription factors, allowing their recognition by the CSN and their subsequent degradation by the $26 \mathrm{~S}$ proteasome (Yi and Deng, 2005; Henriques et al., 2009; Lau and Deng, 2010). In this process, COP1 acts as the central integrator of light responses downstream of photoreceptors (Yi and Deng, 2005; Lau and Deng, 2010).

The plant hormone auxin (indole-3-acetic acid; IAA) plays a major role in morphogenesis and mediates some of the growth responses to light, including hypocotyl elongation, apical hook maintenance, phototropic responses and the shade avoidance syndrome (Boerjan et al., 1995; Zhao et al., 2001; Stepanova et al., 2008; Esmon et al., 2006; Tao et al., 2008). Auxin action depends on a tightly regulated distribution across the plant. Auxin is transported from cell to cell in a polar fashion through the concerted action of several carrier proteins (Friml, 2010). Among them, the PIN-FORMED (PIN) efflux carriers control the direction of auxin flux through their polar localization onto the plasma membrane (PM), thereby promoting the establishment and subsequent maintenance of instructive auxin gradients (Wisniewska et al., 2006; Benková et al., 2003; Friml et al., 2003). In lightgrown seedlings, auxin synthesized in young leaf primordia and transported to the roots has been shown to promote the emergence of lateral root primordia (Bhalerao et al., 2002; Salisbury et al., 2007). This phenomenon is inhibited in etiolated seedlings, leading to the hypothesis that light influences lateral root formation through an effect on shoot-to-root polar auxin transport (PAT) (Bhalerao et al., 2002). Moreover, recent data show that darkness rapidly triggers a change in the intracellular distribution of several PIN 
proteins in the root apical meristem (RAM), which was proposed to lead to a local alteration of auxin transport and to a decrease in root growth (Laxmi et al., 2008). In the case of PIN2, strong evidence indicates that this occurs through a decrease in PIN2 protein levels at the PM that is triggered by degradation through vacuolar targeting of the protein, similarly to gravitropic responses (Kleine-Vehn et al., 2008; Leitner et al., 2012).

Although these findings suggest that modulation of root growth by light might be achieved by influencing local auxin fluxes in the root tip, how this regulation is achieved is entirely unknown. In natural environments, roots are not directly exposed to sunlight, being buried deep down in the soil and how light can directly signal to the root tip remains to be established. Here, we report a novel long-distance mechanism by which light coordinates root growth with shoot development through COP1-mediated regulation of PIN1 transcription in the shoot and of PIN1 and PIN2 intracellular distribution in the root, and identify auxin as a key long-distance signal in light-regulated development.

\section{MATERIALS AND METHODS} Plant materials and growth conditions

Arabidopsis Col-0 ecotype was used as wild type (WT) unless otherwise noted. Previously published lines are described in supplementary material Table S1. CLV3 $>>$ iaaL lines were generated by transforming the CLV3:AlcR line (Deveaux et al., 2003) with the AlcA:iaaL binary plasmid using the floral dipping method (Clough and Bent, 1998). Seedlings were germinated on soil (covered with a thin layer of sand to shield roots from light) or on vertical half-strength MS agar plates at $22^{\circ} \mathrm{C}$, under long-day conditions (16 hours light/8 hours darkness) or continuous darkness.

\section{Chemical treatments}

N-1-naphthylphthalamic acid (NPA) applications on hypocotyls were performed as described (Geldner et al., 2004), during 3 days in light or 4 days in darkness. For lactacystin treatments, 5-day-old seedlings were incubated for 4 hours, in light or in darkness, with $50 \mu \mathrm{M}$ lactacystin or DMSO as mock. For ethanol induction, CLV3:AlcR and CLV3 $>>$ iaaL seeds were germinated and grown on half-strength MS agar plates containing $0.1 \%$ ethanol.

\section{Phenotypic and expression analyses}

Seedlings were imaged with a Nikon Coolpix 990 camera and root length was measured using ImageJ (http://rsbweb.nih.gov/ij/). Quantitative analyses of RAMs were performed as previously described (Dello Ioio et al., 2007).

$\beta$-Glucuronidase (GUS) staining was performed as previously described (Scarpella et al., 2004). Images were taken with a Jenoptik ProgRes C10 camera mounted either on a Zeiss Axioskop 2+ microscope equipped with Nomarski optics or on a Leica MZ12 stereomicroscope.

RNA extraction and quantitative reverse transcription PCR (qRT-PCR) were performed as previously described using the ACTIN 2 gene as a reference (Ciarbelli et al., 2008) and analyzed according to Pfaffl (Pfaffl, 2001). Primer sequences and Universal Probe Library (UPL) probes are given in the supplementary material Table S2.

Epifluorescence images were taken with a Leica M205FA stereomicroscope or an Axioskop 2+ microscope and Leica DFC310 FX or ProgRes C10 camera, respectively. Confocal imaging was performed using Zeiss LSM510 or Leica TCS SP5 microscopes. Propidium iodide (PI; $10 \mu \mathrm{g} / \mathrm{ml}$ in water) was used to counterstain root cells. FM4-64 staining was performed as previously described (Xu and Scheres, 2005). For fluorescence quantification, the same confocal settings were used to compare different genotypes/treatments. All the samples from a single experiment were analyzed serially on the same day. ImageJ was used to quantify fluorescence intensities.

Statistical analyses (pairwise two-tailed Student's $t$-test) were performed using Microsoft Excel. Correction for multiple testing was carried out using the Bonferroni correction.

\section{RESULTS \\ Darkness reduces root growth in a reversible manner}

To gain insight into how root growth is controlled by light, we compared the root development of light-grown and dark-grown (etiolated) Arabidopsis seedlings. Etiolated seedlings had shorter roots than light-grown controls from 6 days after plating on culture dishes (Fig. 1A,B), indicating reduced root growth in darkness. To establish whether this is due to alterations in cell expansion and/or proliferation, we measured the length of differentiated epidermal cells and analyzed the size of the RAM. We found no significant difference in epidermal cell length between light-grown and etiolated seedlings at 4 days after germination and only a slight difference at 12 days (Fig. 1D). However, we observed a strong reduction of the RAM size in darkness (Fig. 1C,E) that was not due to changes in meristem patterning (supplementary material Fig. S1), suggesting that only cell proliferation is inhibited in darkness. We confirmed this by using the CYCB1;1:GUS cell proliferation marker (Colón-Carmona et al., 1999) (Fig. 1F). This inhibition is fully reversible as RAM cell proliferation and root growth was recovered upon light exposure of etiolated seedlings (supplementary material Fig. S2).

\section{Signals from the shoot are involved in adapting root growth to light conditions}

The effect of dark treatments on PIN protein intracellular distribution in the root tip has led to the hypothesis that root growth might be regulated by light through changes in PIN intracellular localization (Laxmi et al., 2008). Unexpectedly, dark-induced changes in PIN-GFP subcellular localization have also been observed in isolated roots (Laxmi et al., 2008), indicating that removal of the shoot apex does not block root responses to changes in light environments. To establish whether roots underground autonomously perceive light or whether signals from the shoot are involved in this regulation, we used the subcellular localization of PIN1-GFP (Xu et al., 2006) and PIN2-GFP (Xu et al., 2006) driven from their endogenous promoter in soil-grown roots (that are in constant darkness) as a proxy for light-induced changes in root development. When the shoot was exposed to light, we observed association of PIN1-GFP with vacuoles [as confirmed by colocalization with the vacuolar marker mRFP-VAM3 (Ebine et al., 2008)] in addition to its PM localization (Fig. 2A), whereas PIN2GFP was strictly associated with the PM (Fig. 2C). This indicates that, despite the stabilizing effect of darkness on GFP in the lytic vacuoles (Tamura et al., 2003), having the root in darkness is not sufficient to trigger high vacuolar PIN1-GFP or PIN2-GFP fluorescence. We observed a strong vacuolar targeting of both PINGFP markers only when shoots were also placed in darkness (Fig. 2B,D). In these conditions, PIN1-GFP almost completely disappeared from membranes (Fig. 2B), whereas PIN2-GFP membrane association was reduced (Fig. 2D). These data indicate that under physiological conditions the regulation of the intracellular localization of PIN proteins by light is not rootautonomous but rather involves signals from the shoot.

\section{Darkness regulates root growth by impairing shoot-to-root PAT}

The root phenotype of etiolated seedlings closely resembles that of light-grown seedlings in which the auxin supply from the shoot apex was removed by shoot decapitation or inhibited by local application of the PAT inhibitor NPA on the hypocotyl (Grieneisen et al., 2007; Reed et al., 1998). We thus hypothesized an 

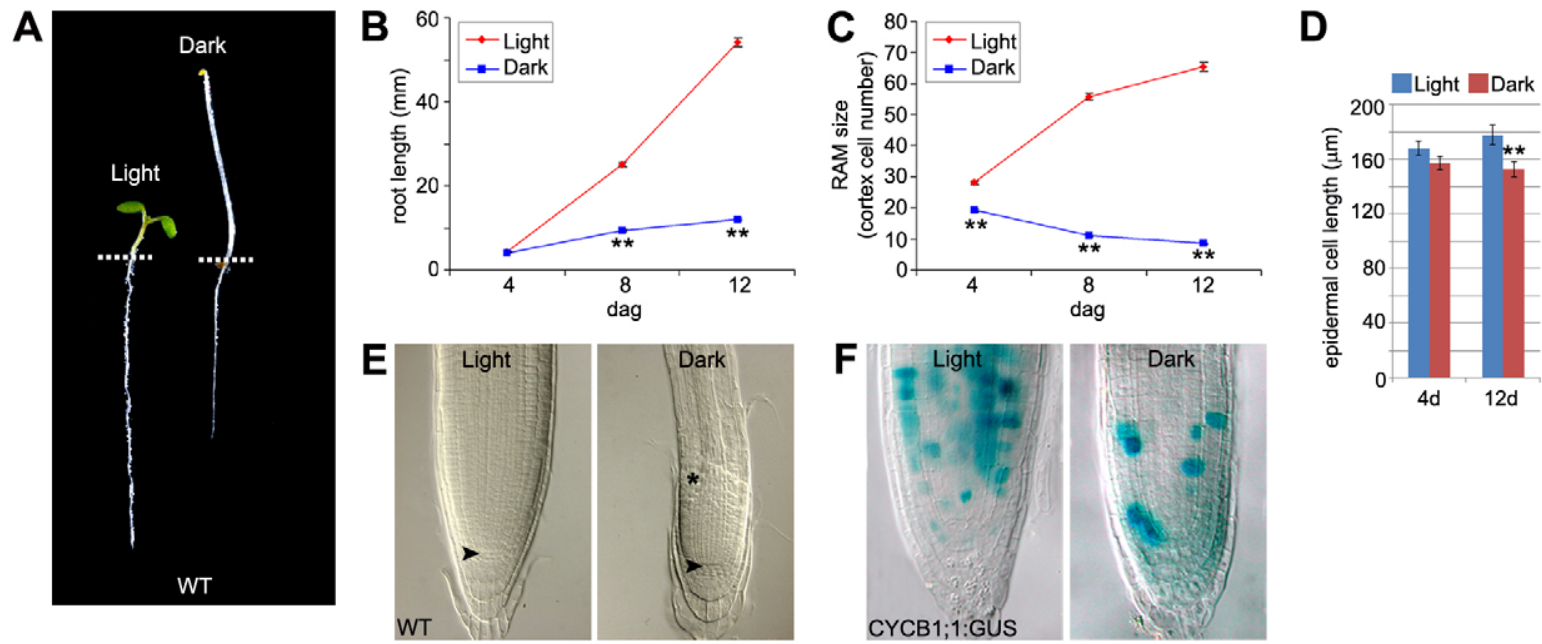

Fig. 1. Light controls root growth in Arabidopsis. (A) Phenotypes of 6-day-old light- and dark-grown WT seedlings. Dotted lines indicate the shoot-root junction. (B,C) Root length (B) and RAM size (C) of WT seedlings grown as indicated. All the differences between light and dark are statistically significant $\left(t\right.$-test, $\left.{ }^{*} P<0.01\right)$. (D) Epidermal cell length in roots of seedlings grown as indicated. ${ }^{*} P<0.01, t$-test. (E) RAM phenotypes of 6-day-old light- and dark-grown seedlings. Asterisk, RAM boundary; arrowheads, quiescent centers. (F) CYCB1;1:GUS expression patterns in RAMs of 4-day-old light- and dark-grown seedlings. Error bars indicate s.e.m. dag, days after germination.

involvement of shoot-derived auxin and shoot-to-root PAT in the control of root growth by light. To verify this hypothesis, we first lowered auxin levels in the shoot apical meristem through ethanolinducible expression of the bacterial gene iaaL, which reduces free auxin by conjugating IAA to Lys (Jensen et al., 1998), in the CLAVATA3 (CLV3) (Fletcher et al., 1999) domain (CLV3>>iaaL). Upon induction, $C L V 3>>i a a L$ seedlings displayed shorter roots and less expanded RAMs compared with CLV3:AlcR controls (Deveaux et al., 2003) when grown in light (Fig. 3A; supplementary material Fig. S3), indicating that shoot-derived auxin is crucial for root growth. By contrast, etiolated CLV3 $>>i a a L$ and CLV3:AlCR seedlings showed no obvious differences after induction (Fig. 3A; supplementary material Fig. S3). Coherently, similar results were also obtained when shoot-toroot PAT was inhibited by local application of NPA on hypocotyls of WT seedlings (Fig. 3B; Fig. 4B,C; supplementary material Fig. S9). Taken together, these data support the idea that darkness inhibits shoot-to-root PAT.

To provide further evidence that shoot-to-root PAT is impaired in darkness, we used the DII-VENUS auxin sensor (Brunoud et al., 2012). DII-VENUS allows detection of dynamically relative changes in auxin distribution with cellular resolution (Brunoud et al., 2012). Whereas DII-VENUS could not be detected in lightgrown hypocotyls (Fig. 3C,D), we observed a gradient of DIIVENUS fluorescence in etiolated hypocotyls, with the highest fluorescence in the basal region of the hypocotyl (Fig. 3E-J). Similarly, DII-VENUS fluorescence was greatly increased in darkgrown RAMs compared with light controls (Fig. 3K,L). These changes were not observed with the mDII-VENUS auxininsensitive version of the sensor (supplementary material Fig. $\mathrm{S} 4 \mathrm{~A}$ ). In addition, the tissue-specific expression along the hypocotyl axis or in the RAMs of GUS translational fusions to the TIR1/AFB1-3 auxin co-receptors (Parry et al., 2009) was not affected by the light conditions (supplementary material Fig. S4B), indicating that the differences in DII-VENUS fluorescence are not due to altered auxin sensitivity in response to light. These results demonstrate a decrease in auxin concentration along the hypocotyl axis and in the RAM. Further strengthening our observations, expression of the synthetic auxin-inducible marker DR5:GUS (Ulmasov et al., 1997) was strongly enhanced in the aerial tissues
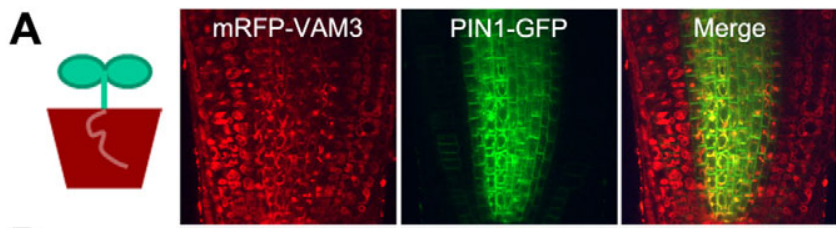

B
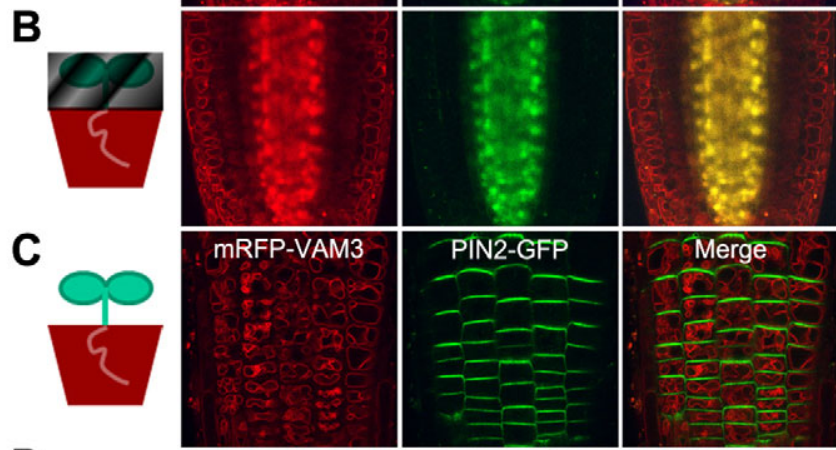

D
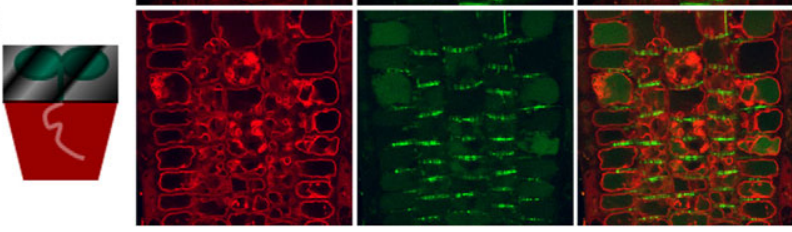

Fig. 2. Light regulates PIN1-GFP and PIN2-GFP intracellular localization in roots of Arabidopsis plants grown on soil.

$(\mathbf{A}, \mathbf{B})$ PIN1-GFP and mRFP-VAM3 expression in the root vascular tissue of 7-day-old plants grown on soil with the shoot in light (A) or in darkness (B). (C,D) PIN2-GFP and mRFP-VAM3 expression in the root epidermis of 7-day-old plants grown on soil with the shoot in light (C) or in darkness (D). Note that the morphology of the vacuoles visualized with mRFP-VAM3 is different between tissues and that it changes with the light conditions. 

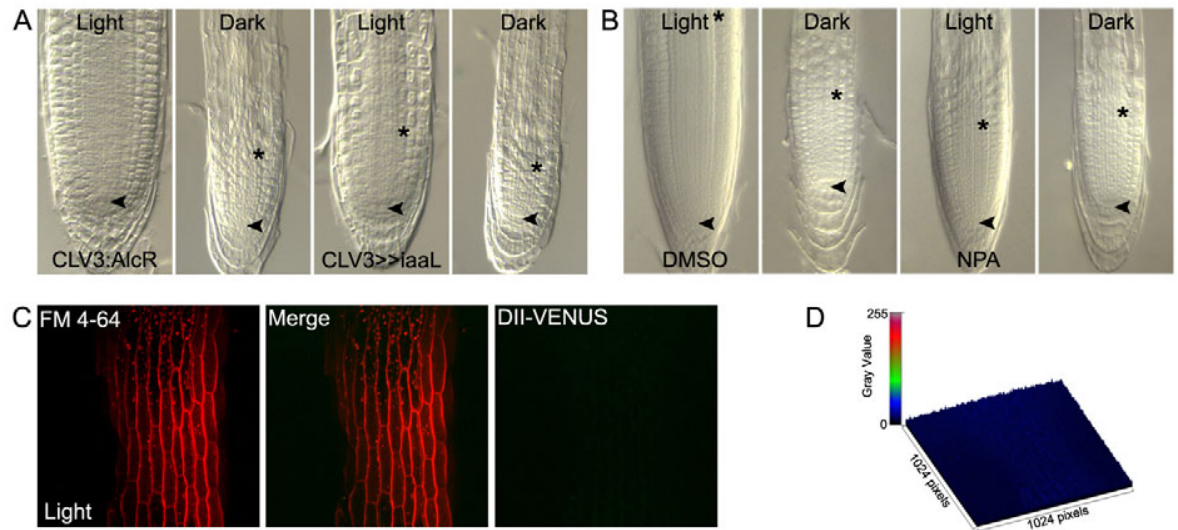

DMSO
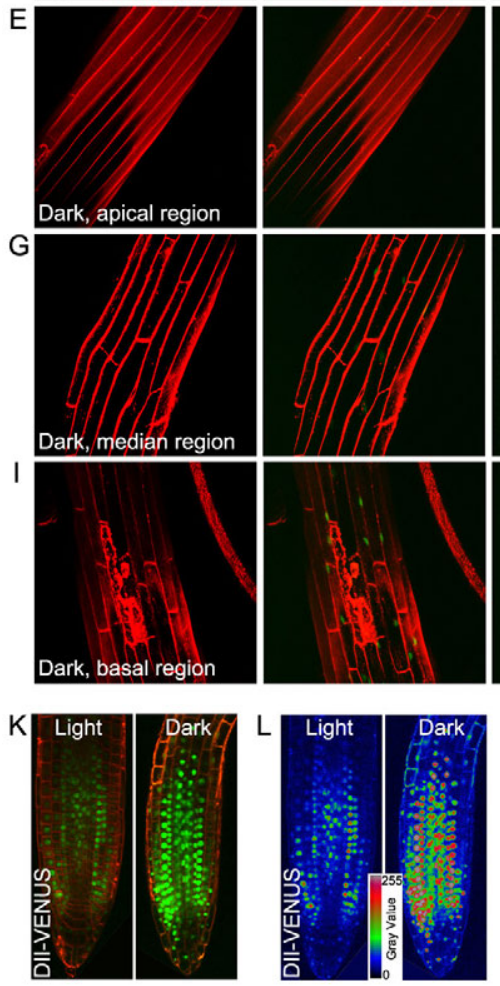

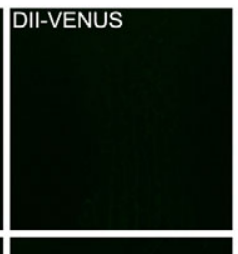

D

$\mathrm{F}$
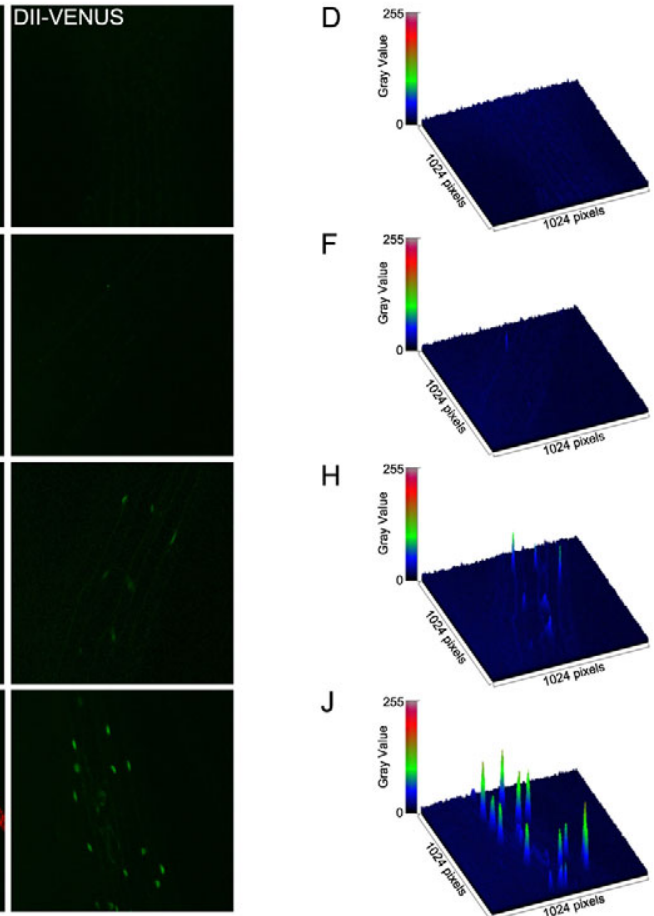

$\mathrm{H}$

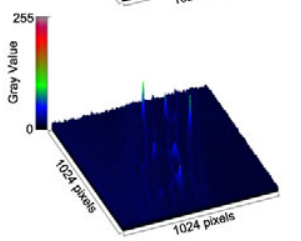

$\mathrm{J}$
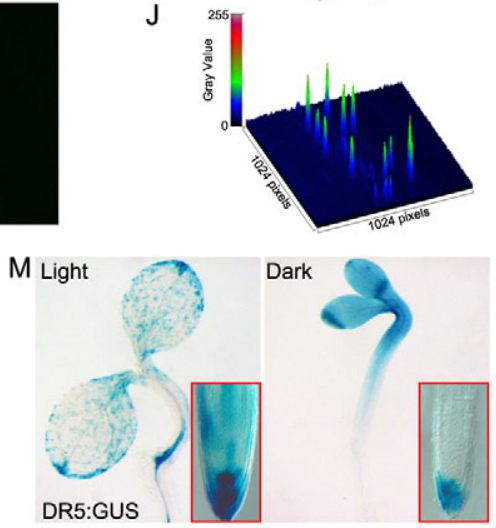

Fig. 3. Light regulates shoot-to-root PAT in Arabidopsis. (A,B) RAM phenotypes of 4day-old light- and dark-grown CLV3:AlcR and CLV3>>iaaL seedlings upon ethanol induction (A) and of 7-day-old light-grown and 9-dayold dark-grown WT seedlings with and without (DMSO) NPA application on the hypocotyls (B). Asterisks, RAM boundaries; arrowheads, quiescent centers. (C,D) DIIVENUS expression (C) and fluorescence intensity (D) in 4-day-old light-grown hypocotyls. Red channel, FM4-64 staining. (E-J) DII-VENUS expression $(E, G, I)$ and fluorescence intensity $(F, H, J)$ in the apical $(E, F)$, median $(\mathrm{G}, \mathrm{H})$ and basal $(\mathrm{I}, \mathrm{J})$ regions of 4-dayold etiolated hypocotyls. Red channel, FM4-64 staining. (K, L) DII-VENUS expression (K) and fluorescence intensity (L) in RAMs of 4-day-old light- and dark-grown seedlings. Red channel, FM4-64 staining; inset, false color scale. (M) DR5:GUS expression in shoots and RAMs (insets) of 4-day-old light- and dark-grown seedlings. of etiolated seedlings, just above the apical hook, and markedly reduced in the RAM compared with light-grown controls (Fig. $3 \mathrm{M})$. Our data thus suggest a decrease in shoot-to-root PAT in etiolated hypocotyls that leads to auxin depletion in the RAM and concurrent accumulation in the shoot apex.

\section{Darkness regulates PIN1 transcription in the hypocotyl}

To identify the mechanisms underlying the changes in shoot-to-root PAT, we next investigated whether light could modulate the activity of the PIN family of auxin efflux carriers in the hypocotyl. Among the PIN genes, only PIN1, PIN3 and PIN7 have been reported to be expressed in hypocotyls (Blakeslee et al., 2007; Vieten et al., 2005). We therefore analyzed the expression patterns of PIN1:GUS, PIN3:GUS and PIN7:PIN7-GUS (Vieten et al., 2005) in light-grown and etiolated seedlings. PIN1:GUS expression was dramatically reduced in the hypocotyls of etiolated seedlings relative to the strong staining observed in light-grown seedlings (Fig. 4A). By contrast, GUS expression in both PIN3:GUS and PIN7:PIN7-GUS hypocotyls, albeit lower compared with light-grown seedlings, was largely retained in darkness (Fig. 4A). Similar results were obtained when PIN1, PIN3 and PIN7 expression was analyzed using qRTPCR, but a PIN2 control showed no regulation by light (supplementary material Fig. S5A,B). In addition, we observed that the PIN1-GFP fluorescence also decreased in dark-grown PIN1:PIN1-GFP hypocotyls, indicating reduced PIN1 protein levels (supplementary material Fig. S5C). Thus, our results suggest that light might control shoot-to-root PAT mainly through modulating PIN1 transcription in the hypocotyl.

\section{PIN1-dependent shoot-to-root PAT is crucial for root growth}

To establish a role for PIN1-dependent shoot-to-root PAT in root growth, we applied NPA locally to the hypocotyls of light-grown WT and pin 1-6 (Vernoux et al., 2000) seedlings, and analyzed their root phenotypes. NPA strongly reduced root length and RAM size in WT plants (Fig. 4B,C), whereas in pin 1 mutants RAM size was unaffected and root length was only mildly reduced (Fig. 4B,C). RAM size of untreated pin 1 mutants was nearly identical to that of NPA-treated WT plants (Fig. 4C), 
A

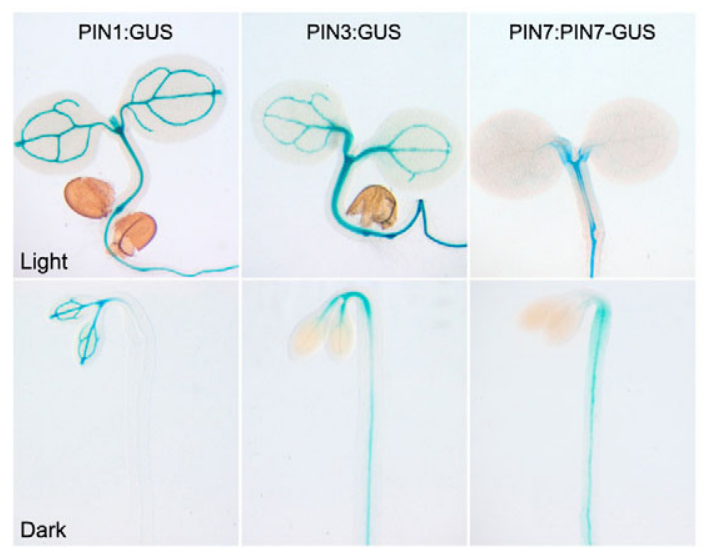

B

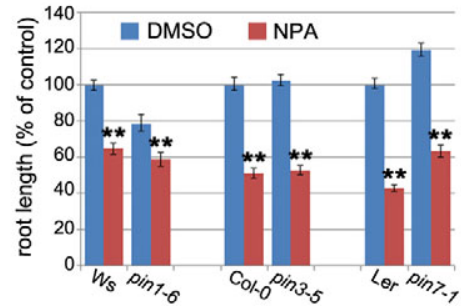

C

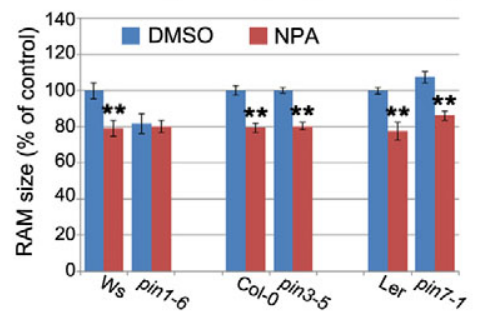

$\mathbf{F}$

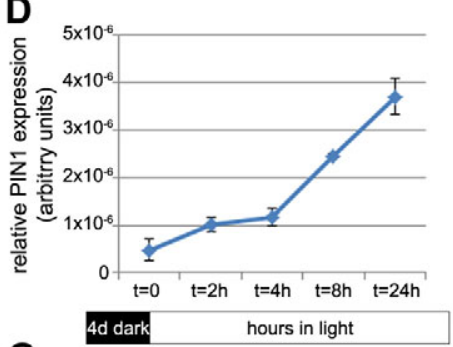

E

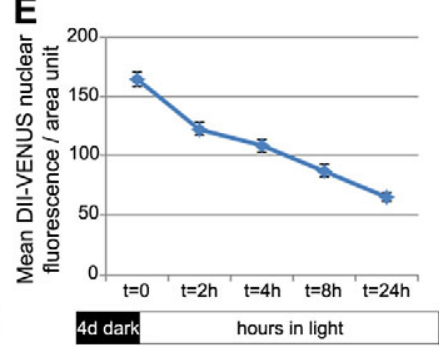

Fig. 4. PIN1 expression, auxin levels and root growth phenotypes during de-etiolation are closely correlated. (A) PIN1:GUS, PIN3:GUS and PIN7:PIN7-GUS expression in shoots of 4-dayold light- and dark-grown Arabidopsis seedlings.

$(\mathbf{B}, \mathbf{C})$ Root length $(B)$ and RAM size (C) of light-grown pin 1-6, pin3-5 and pin7-1 mutants and their WT controls (Ws, Col-0 and Ler, respectively) with or without (DMSO) NPA

application on the hypocotyl. Error bars indicate s.e.m.

$* * P<0.01$, $t$-test. (D) PIN1 expression, determined by qRT$P C R$, in shoot tissues of seedlings grown as indicated. Error bars indicate s.d. (E-G) DIIVENUS expression (G), mean nuclear fluorescence (E) and number of DIl-expressing nuclei (F) in basal hypocotyls of seedlings grown as indicated. White lines indicate hypocotyl boundaries. Error bars indicate s.e.m. $(\mathbf{H}, \mathbf{I})$ Root length $(H)$ and RAM size (I) of WT and pin 1-6 grown for 4 days in darkness and then either exposed to light, or kept in darkness for the following 4 days. In $\mathrm{H}$, ${ }^{*} P P<0.01$, $t$-test. In I, asterisks, RAM boundaries; arrowheads, quiescent centers.

$\mathrm{H}$

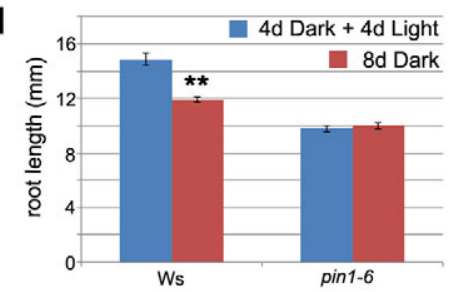

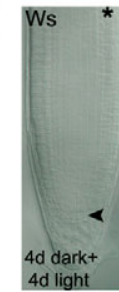

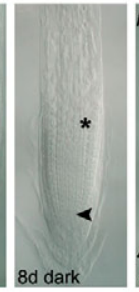

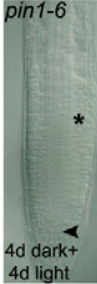

\begin{abstract}
$=2$
\end{abstract}

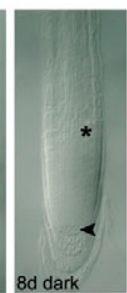

supporting a key role for PIN1-dependent shoot-to-root PAT in the regulation of RAM size. By contrast, pin3-5 and pin 7-1 (Benková et al., 2003) mutants had a WT-like response to NPA (Fig. 4B,C), suggesting that PIN3 and PIN7 play a negligible role in the light-regulation of root growth.

To provide further evidence for the role of PIN1 transcriptional regulation in the hypocotyl, we analyzed by qRT-PCR its expression in hypocotyls after shifting etiolated seedlings back into light and observed a rapid and continuous induction of PIN1 over a 24 hour period (Fig. 4D). This induction correlated with a progressive decrease of DII-VENUS fluorescence (suggesting an increase in auxin concentration) in the basal region of the hypocotyl (Fig. 4E-G). In addition, we observed that the pin1 mutants could not recover root growth and RAM size during deetiolation, demonstrating that PIN1 is essential for this process (Fig. 4H,I). Taken together, our data indicate that the regulation of PIN1 transcription by light is crucial for controlling shoot-to-root PAT and root growth in response to light.

\section{Genetic evidence indicates that PIN2 is also involved in the control of root growth by light}

The effect of darkness on the intracellular distribution of PINs suggests that these proteins might also act directly in the RAM to regulate root growth in different light conditions. Previous observations have shown that at least PIN1, PIN2, PIN3, PIN4 and PIN7 are expressed in the RAM where they act partially redundantly to modulate root growth in light (Blilou et al., 2005). We thus analyzed root phenotypes of single and multiple pin mutants grown in light and in darkness. As previously observed (Blilou et al., 2005), light-grown pin2 (eir1-1) (Luschnig et al., 1998) mutants showed the most severe reduction of RAM size (Fig. 5B; supplementary material Fig. S6), supporting a key-role for PIN2 in regulating RAM cell proliferation (Blilou et al., 2005). However, all the etiolated pin single mutants exhibited reduced root length and RAM size, suggesting possible redundancy between $P I N$ genes in this process (Fig. 5A,B). Among higher order mutants, pin1 pin2 double mutant (Blilou et al., 2005) displayed 

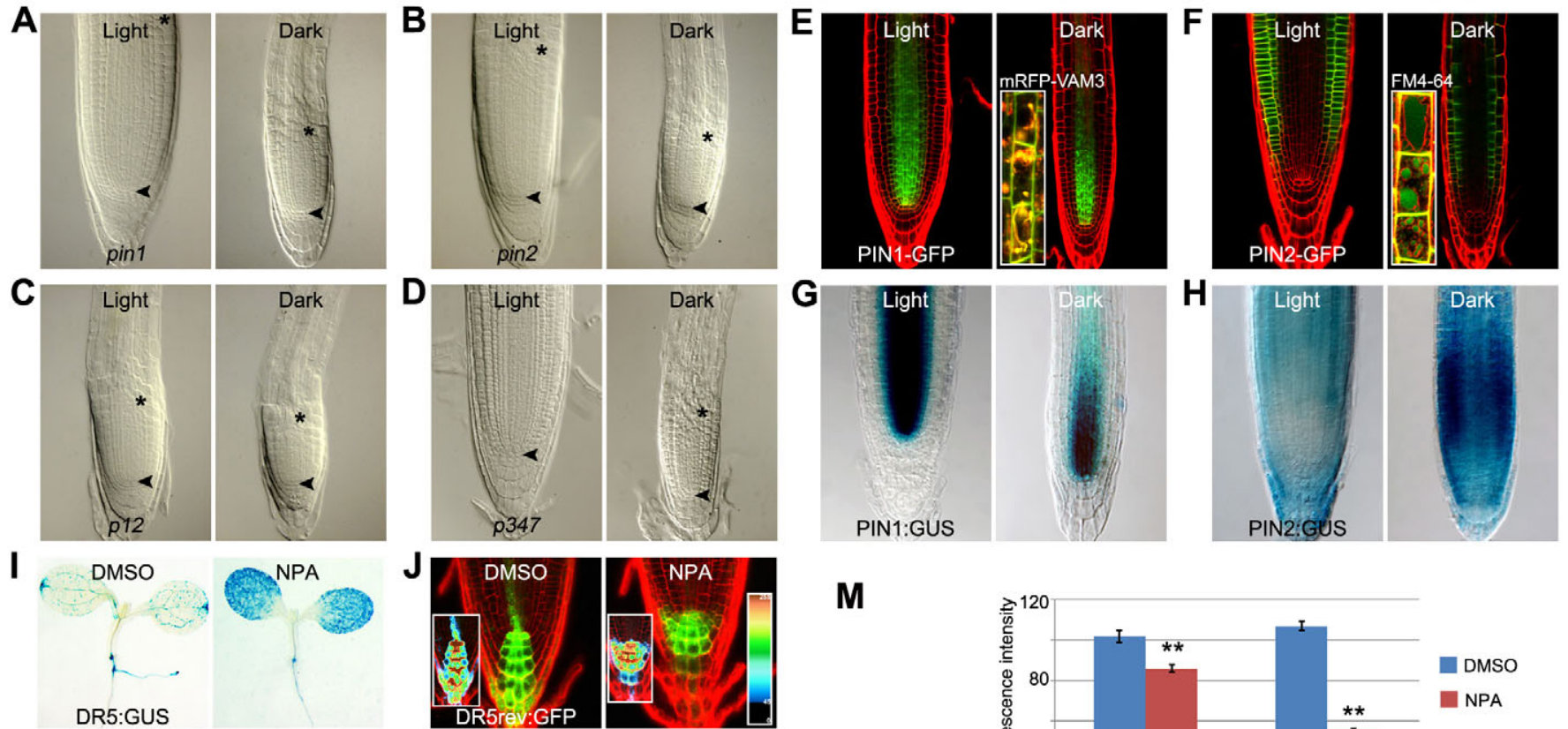

M
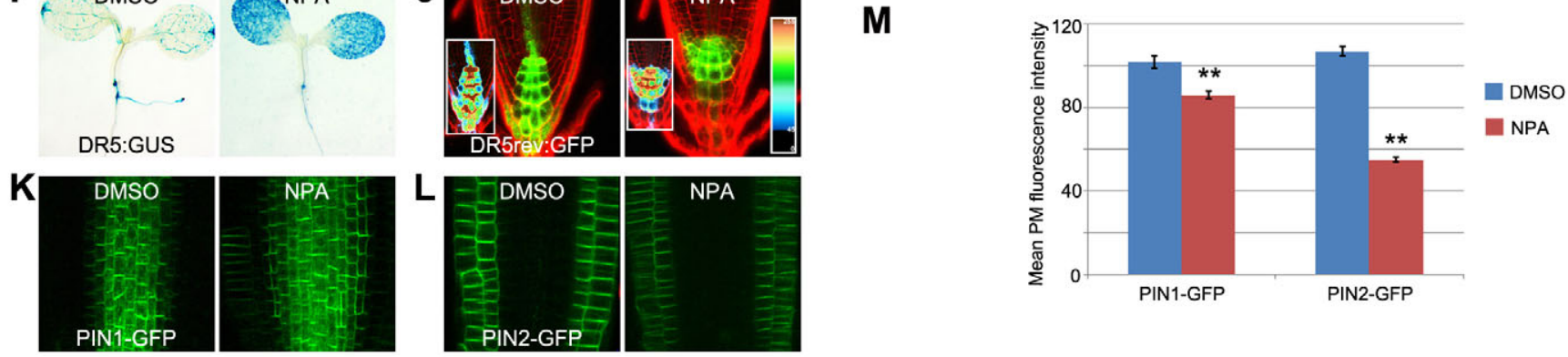

Fig. 5. Light controls root growth through PIN1 and PIN2. (A-D) RAM phenotypes of 6-day-old light- and dark-grown pin1 (A), pin2 (B), pin1 pin2 (p12) (C) and pin3 pin4 pin7 (p347) (D) Arabidopsis seedlings. Asterisks, RAM boundaries; arrowheads, quiescent centers. (E-H) PIN1:PIN1-GFP (E), PIN2:PIN2-GFP (F), PIN1:GUS (G) and PIN2:GUS (H) expression in 6-day-old RAMs. Inset in E: colocalization of PIN1-GFP (green) and mRFP-VAM3 (red) in etiolated roots; inset in F: colocalization of PIN2-GFP (green) and FM4-64 staining (red). (I-L) DR5:GUS (I), DR5rev:GFP (J), PIN1:PIN1-GFP (K) and PIN2:PIN2-GFP (L) expression in light-grown seedlings with and without (DMSO) NPA application on the hypocotyls. Insets in $J$ depict pseudocolorimetric quantifications of GFP intensities. (M) Quantification of PM PIN1-GFP and PIN2-GFP levels in RAMs grown as shown in K and L, respectively. Error bars indicate s.e.m. ${ }^{*} P<0.01, t$-test.

severely reduced root length and RAM size in both light and dark conditions (Blilou et al., 2005; Vieten et al., 2005) (Fig. 5C; supplementary material Fig. S6). By contrast, pin3 pin4 pin7 triple mutants (Blilou et al., 2005) were indistinguishable from the WT regardless of the light condition (Fig. 5D; supplementary material Fig. S6). Together, our results reveal that PIN1 and PIN2 (but not PIN3, PIN4 and PIN7) are crucial regulators of root growth in response to light conditions. Our data further suggests that rootautonomous modulation of PAT through changes in both PIN1 and PIN2 activity might also control root growth in response to light.

\section{PIN1-dependent shoot-to-root PAT modulates PIN1 and PIN2 intracellular distribution in the root tip}

As demonstrated previously, darkness induces drastic changes in PIN1 and PIN2 intracellular localization in agar-grown roots (Laxmi et al., 2008; Kleine-Vehn et al., 2008) (Fig. 5E,F). This leads to a reduction in the abundance of PM-localized PIN1 and PIN2 and of the total pool of protein in the case of PIN2 (Fig. 5E,F; Fig. 6G,H). Transcription was unchanged for PIN1 and increased for PIN2, suggesting that these changes do not result directly from modifications in the transcription of the two genes (Fig. 5G,H). Because auxin can modulate PIN intracellular localization and stability (Paciorek et al., 2005; Sauer et al., 2006; Abas et al., 2006; Sieberer et al., 2000; Vieten et al., 2005), we wondered whether reduced shoot-to-root PAT in darkness could cause these changes. To test this, we inhibited shoot-to-root PAT in light-grown $D R 5: G U S$, DR5rev:GFP (Friml et al., 2003), PIN1:PIN1-GFP and PIN2:PIN2$G F P$ seedlings using local NPA application to the hypocotyl (Fig. 5I$\mathrm{M})$. This treatment caused a dramatic reduction of the PM abundance of PIN2-GFP, and to a lesser extent of PIN1-GFP, in the RAM compared with the untreated controls (Fig. 5K-M). The same treatment also led to enhanced DR5:GUS expression in the cotyledons (Fig. 5I) and lateral expansion of the DR5rev:GFP domain in the RAM (Fig. 5J), resembling that observed during etiolation (Fig. 3M) or in light-grown pin 2 mutant (Ottenschläger et al., 2003). Our results support a scenario in which dark-induced reduction in PIN1 activity in the hypocotyl leads to lower auxin levels in the root, which in turn promotes a reduction in PIN1 and PIN2 levels at the PM, thus impairing root growth. Consistently, NPA application at the hypocotyls of pin2 seedlings triggered a reduction in root length but did not cause any further inhibition of RAM size (supplementary material Fig. S7), providing additional evidence that shoot-derived auxin requires PIN2 function in the root tip to control the RAM size and root growth in response to light.

\section{Mutations in COP1 improve root growth in darkness}

Having established that light conditions act by modulating auxin transport both in the hypocotyl and in the root tip, we next investigated the molecular pathways involved in this regulation. To 

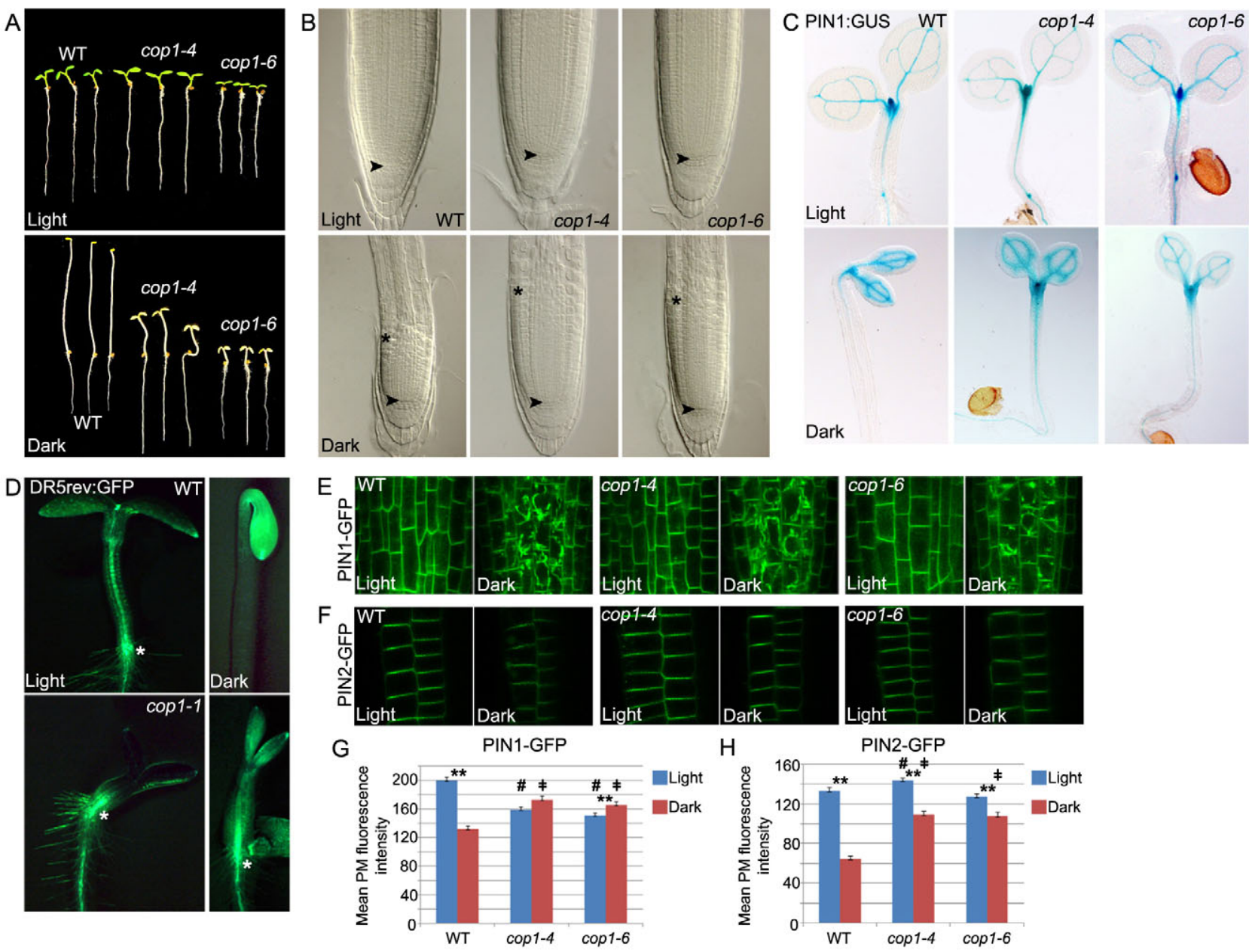

Fig. 6. COP1 regulates root growth, PIN1 expression and PIN1-GFP and PIN2-GFP in response to change in light conditions. (A) Seedling phenotypes of 6-day-old light- and dark-grown WT, cop1-4 and cop1-6 Arabidopsis plants. (B) RAM phenotypes of 6-day-old light- and dark-grown WT, cop 1-4 and cop1-6 plants. Asterisks, RAM boundaries; arrowheads, quiescent centers. (C) PIN1:GUS expression in shoots of 6-day-old lightand dark-grown WT, cop 1-4 and cop1-6 plants. (D) DR5rev:GFP expression in 6-day-old WT and cop1-1 seedlings. Asterisks, shoot-root junctions. (E,F) PIN1-GFP (E) and PIN2-GFP (F) localization in RAMs of 6-day-old light- and dark-grown WT, cop1-4 and cop1-6 plants. (G,H) Quantification of PM PIN1-GFP (G) and PIN2-GFP (H) levels in WT, cop1-4 and cop1-6 plants grown as shown in E,F. Significant differences are indicated $(P<0.05$ with Bonferroni correction): ${ }^{* *}$, dark versus light; \# or ${ }^{\ddagger}$, genotype versus WT in the light or the dark, respectively. Error bars indicate s.e.m.

this end, we analyzed the root phenotypes of the cop1-4 and cop16 (McNellis et al., 1994) mutant alleles, which have hypomorphic mutations in the COP1 gene, which encodes a master regulator of light-controlled shoot development (Yi and Deng, 2005). Compared with WT, copl mutants displayed reduced root length in light but longer roots in darkness (Fig. 6A; supplementary material Fig. S8A,C), suggesting a role for COP1 in both light and dark conditions. Strikingly, all the cop 1 mutants displayed more expanded RAMs compared with WT controls (Fig. 6B; supplementary material Fig. S8B,D) regardless of the light conditions. These phenotypes suggest that COP1 plays a key role in mediating the regulation of root growth in response to light.

\section{COP1 acts together with both PIN1 and PIN2 to regulate root growth}

To test whether COP1 controls root growth in response to light by regulating PIN1 and PIN2, we next generated cop1-4 pin1-1, cop1-6 pin1-1, cop1-4 pin2 and cop1-6 pin2 double mutant combinations. The pin1-1 mutation significantly affected the cop1 root phenotype. Both cop1-4 pin1-1 and cop1-6 pin1-1 displayed reduced root length and smaller RAM size compared with the parental cop1 alleles both in light and in darkness (supplementary material Fig. S8A,B). However, cop1 pin 1 double mutants always showed longer roots and more expanded RAMs compared with pin1-1 in darkness (supplementary material Fig. S8A,B), indicating that combining the copl and pin 1 mutations partially rescue the capacity of the plant to adapt root growth to light conditions. Similarly to cop 1 pin 1 double mutants, both cop 1-4 pin2 and cop 1-6 pin 2 mutants displayed shorter roots and smaller RAMs compared with the parental cop1 alleles regardless of the light conditions (supplementary material Fig. S8C,D), whereas dark-grown cop1 pin2 root phenotypes were not as severe as those of the pin2 mutant (supplementary material Fig. S8C,D). The combination of the pin 2 and cop 1 mutations thus also rescues, in part, the capacity of the plant to adapt root growth to light conditions, indicating that COP1, PIN1 and PIN2 participate together in the regulation of root growth by light. 


\section{COP1 regulates PIN1 transcription in the hypocotyl to control PIN1-dependent shoot-to- root PAT}

We then investigated whether COP1 could control the regulation of PIN1 transcription in the hypocotyl and analyzed PIN1:GUS expression in light- and dark-grown cop1-4 and cop 1-6 hypocotyls. Whereas PIN1:GUS expression was dramatically reduced in the hypocotyls of etiolated WT seedlings compared with light-grown controls (Fig. 6C), expression of the transgene was retained in the hypocotyls of dark-grown copl mutants, resembling what we observed in light-grown WT and copl seedlings (Fig. 6C). COP1 might thus be involved in downregulating PIN1 transcription in the hypocotyls of darkgrown seedlings and in the inhibition of shoot-to-root PAT and root growth. To confirm this hypothesis, we introgressed DR5rev:GFP in a copl background. We observed that, whereas DR5rev:GFP expression was restricted to the apical organs and markedly reduced in the hypocotyl below the apical hook region in WT (Fig. 6D), its expression was retained in the hypocotyls of dark-grown cop 1-1 seedlings, similarly to light-grown WT seedlings (Fig. 6D). This further substantiates a role for COP1 in the regulation of PIN1-dependent shoot-to-root PAT.

\section{COP1 modulates PIN1 and PIN2 intracellular distribution in the root}

As COP1 interacts genetically with both PIN1 and PIN2, it is unlikely to act only through the regulation of PIN1 transcription in the hypocotyl in response to light and might also play additional roles in the RAM. To confirm this, we applied NPA to the hypocotyls of dark-grown plants with WT and copl alleles, to test whether the inhibition of shoot-to-root PAT is sufficient to impair root growth and RAM size in the mutants. In line with the downregulation of PIN1 expression in darkness, NPA-treated WT seedlings did not display any difference in root length and in RAM size compared with untreated controls. By contrast, NPA strongly inhibited root length in dark-grown copl mutants, but their RAM size was unchanged compared with untreated controls (supplementary material Fig. S9A,B). It should be noted that local application of NPA inhibited hypocotyl elongation in both WT and cop1 mutants (supplementary material Fig. S9C), indicating that NPA effectively inhibits shoot-to-root PAT in copl hypocotyls. Together, our results indicates that COP1 might also play a role locally in the root to regulate RAM size.

We next tested whether COP1 also regulates the intracellular localization of PIN1 and PIN2 in the RAM in response to light. To do so, we compared PIN1-GFP and PIN2-GFP PM abundance and intracellular distribution in RAMs of WT and copl plants in both light and dark conditions. The copl mutation did not affect the changes in intracellular localization of PIN1-GFP induced by darkness. However, quantification of PM fluorescence showed that, unlike WT roots (Fig. 6E,G), the PM abundance of PIN1-GFP in cop 1-4 and cop1-6 roots (Fig. 6E,G) was not reduced by darkness. Dark-induced accumulation of PIN2-GFP in the vacuolar lumen (Laxmi et al., 2008) was, by contrast, blocked by mutations in COP1 (Fig. 6F). Similarly to PIN1-GFP, in darkness both cop1-4 and cop1-6 had a higher intensity of PIN2-GFP at the PM than did WT controls (Fig. 6F,H). We thus conclude that, in the RAM, COP1 regulates the decrease in PM-associated PIN1 and PIN2 and also the changes in intracellular localization of PIN2 triggered by darkness. In addition, in light-grown seedlings, NPA application at the hypocotyls of the cop 1-4 mutant did not cause any reduction in the amounts of PM-localized PIN1-GFP and PIN2-GFP, as observed for WT plants (supplementary material Fig. S10), further supporting a root-autonomous role of COP1 in the regulation of PIN1 and PIN2 intracellular localization and, thus, of RAM size.

\section{COP1 regulates PIN2 stability}

The changes in PIN2 intracellular distribution in darkness have been proposed to result from a higher ubiquitination rate of PIN2, leading to degradation in vacuoles (Kleine-Vehn et al., 2008; Leitner et al., 2012). As previously reported using MG132 (Abas et al., 2006; Laxmi et al., 2008), specific inhibition of proteasome activity using lactacystin blocked PIN2-GFP targeting to the vacuole in darkness (supplementary material Fig. S11). This confirms an involvement of the ubiquitin pathway in dark-mediated PIN2 degradation. However, this treatment had no visible effect on the intracellular distribution of PIN1-GFP, suggesting that PIN1 might be regulated differently (supplementary material Fig. S11).

Given that PIN2 degradation is also essential for root gravitropism (Abas et al., 2006), we reasoned that a role for COP1 in the regulation of this developmental response would provide independent evidence for its involvement in the regulation of PIN2 stability. cop 1 mutants indeed demonstrated a reduced capacity to reorientate root growth in response to a change in the gravity vector (Fig. 7A). In addition, copl mutations also interfered with the degradation of PIN2-GFP on the upper side of gravistimulated roots (Abas et al., 2006) (Fig. 7B-D). Our data thus demonstrate that COP1 is involved in regulating PIN2 stability during gravitropism, further supporting a similar role in the RAM in response to changes in light conditions.

\section{DISCUSSION}

Although they are not directly exposed to light in most plant species, light influences the growth characteristics of roots (Feldman, 1984; Halliday et al., 2009). How root growth is controlled by light and integrated with developmental responses in the above-ground organs to shape the overall plant architecture is still largely unknown. Here, we provide strong evidence that light regulates root growth by modulating shoot-to-root PAT and identify auxin as a crucial long-distance signal in the regulation of root growth by light. We show that chemical inhibition of PAT in the hypocotyl, or decreasing shoot-derived auxin by genetic approaches, mimic the effect of darkness on root growth. Our results using the DII-VENUS and DR5 auxin signaling sensors (Brunoud et al., 2012; Ulmasov et al., 1997; Friml et al., 2003), further suggest that dark-induced inhibition of shoot-to-root PAT in the hypocotyl results in auxin accumulation in the shoot apex and in a reduction in auxin levels at the base of the hypocotyl and in the root apex. This reversibly impairs RAM cell proliferation and root elongation in a manner that allows recovery of root growth upon re-exposure to light. The possible accumulation of auxin in aerial tissues resulting from the inhibition of shoot-to-root PAT might also participate in the regulation of light-regulated auxin responses in the shoot, such as the maintenance of the apical hook (Stepanova et al., 2008; Zádníková et al., 2010), and hence to the coordination of growth responses at the level of the entire organism.

At the molecular level, we demonstrate that darkness leads to a strong reduction in PIN1 expression in the hypocotyl that might lead to a decrease in shoot-to-root PAT (although direct measurements of auxin transport would be necessary to definitively prove this). Conversely, PIN1 is induced during de-etiolation with kinetics that correlate with a progressive increase in auxin levels at the base of the hypocotyl as suggested by DII-VENUS 


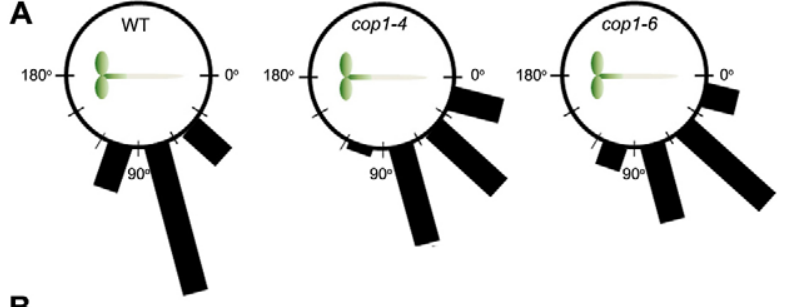

B
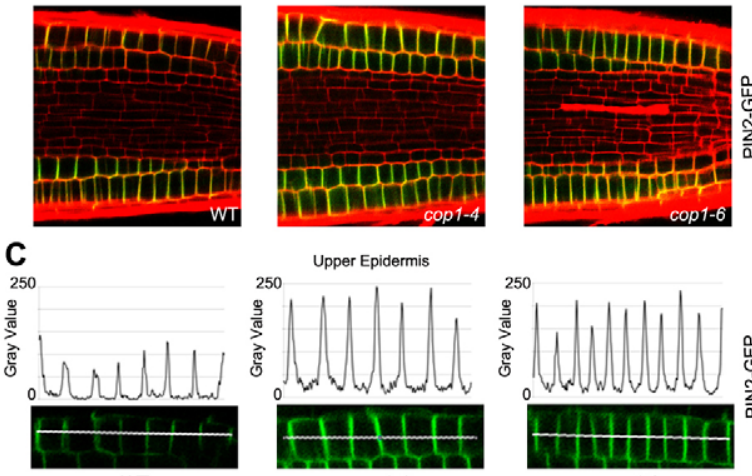

WT

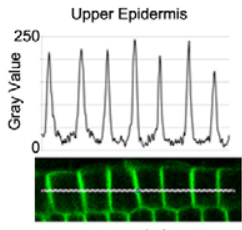

cop 1-4
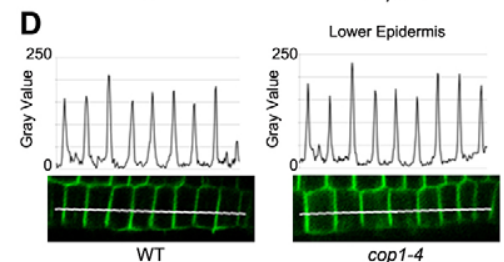

cop1-4
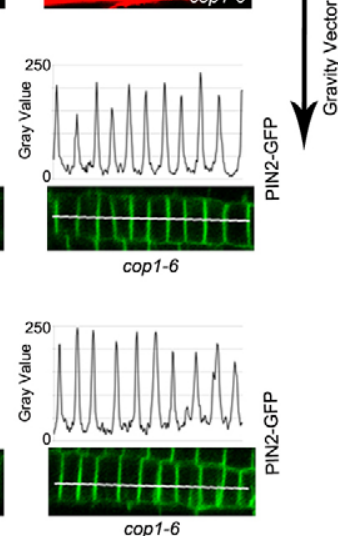

Fig. 7. Impaired gravitropic responses in cop1 mutants. (A,B) Root reorientation (A) and PIN2-GFP expression (B) in WT, cop1-4 and cop1-6 Arabidopsis plants after a $90^{\circ}$ shift of the gravity vector for 8 hours (A) or 3 hours (B). The length of black bars in A represents the relative frequency of the corresponding classes of angles. Red channel in $B$, propidium iodide. (C,D) Quantification of PM PIN2-GFP levels in upper (C) and lower (D) epidermis of roots grown as described for B. Graphs represent PM PIN2-GFP fluorescence intensity calculated along the white lines shown below.

fluorescence. Changes in PIN1 transcription have also been reported using genome-wide expression analysis (López-Juez et al., 2008) and light might then control shoot-to-root PAT in the hypocotyl primarily by regulating PIN1 transcription. This conclusion is further supported by the observation that pin1 mutants have reduced root length and RAM defects identical to those of plants with inhibited shoot-to-root PAT and that the pin1 mutation severely impairs the reversibility of root growth inhibition induced by darkness. Our data thus suggest that PIN1 function in the shoot is not restricted to the inflorescence (Gälweiler et al., 1998; Vernoux et al., 2000; Reinhardt et al., 2003), but is also responsible for the long-distance delivery of shoot-derived auxin to the RAM.

RAM cell proliferation is regulated by PIN-mediated recirculation of shoot-derived auxin across the root apex (Blilou et al., 2005; Grieneisen et al., 2007). Of all the PIN proteins, PIN2 plays a pivotal role in the RAM as it regulates non-redundantly the root basipetal transport of auxin (Blilou et al., 2005). Mutations in PIN2 result in the most severe defects in root growth and RAM proliferation among single pin mutants (Blilou et al., 2005). In roots of etiolated seedlings both in vitro and in soil, the intracellular localization of PIN2-GFP is severely altered, being greatly reduced at the PM and accumulating in the lytic vacuole (Kleine-Vehn et al., 2008; Laxmi et al., 2008) (our data). Furthermore, we have demonstrated that inhibition of shoot-to-root PAT using NPA application on hypocotyls of light-grown seedlings decreases PIN2 levels at the PM in the RAM, and causes root phenotypes similar to those of etiolated seedlings. This suggests that depletion of shoot-derived auxin in darkness is sufficient to decrease PIN2 levels at the PM, a step that might be essential for the inhibition of cell proliferation in the RAM. This role for shoot-derived auxin is further supported by the fact that auxin promotes PIN2 localization to the PM (Paciorek et al., 2005) and by a recent work demonstrating that dark-induced PIN2 targeting to the lytic vacuole can be prevented by exogenous IAA (Rosado et al., 2010).

Besides PIN2, changes in intracellular localization of PIN1, PIN3 and PIN7 in the RAM of dark-grown plants have also been demonstrated (Laxmi et al., 2008) (our data). However, our genetic analysis indicates that, among these, only PIN1 plays a relevant role locally in the RAM. PIN1 contributes to the regulation of cell proliferation together with PIN2, as demonstrated by the increased severity of the root phenotype of pin 1 pin 2 double mutants compared with the single mutants and independently of the light conditions (Blilou et al., 2005; Vieten et al., 2005) (our data). The PM abundance of PIN1 is also reduced in darkness, although to a lesser extent than PIN2. Similarly to PIN2, PIN1 endocytosis is inhibited by auxin (Paciorek et al., 2005), indicating that this reduction in darkness might result from the decrease in shoot-toroot PAT. Dark-mediated changes in PIN1 activity might then function in reducing acropetal transport in etiolated roots. Together with the reduced basipetal flux resulting from higher rates of PIN2 degradation, this could lead to a global decrease in the recirculation of auxin in the RAM, explaining the reduction in cell proliferation and the reversibility of the growth arrest in darkness.

The E3 ubiquitin ligase COP1 has been shown to function as the master regulator of photomorphogenesis (Yi and Deng, 2005). Our data reveal a dual role for COP1 in the regulation of light-mediated root growth, as it regulates both long-distance transport and local RAM fluxes of auxin through different mechanisms. Upon changes in light conditions, COP1 regulates PIN1 expression in the hypocotyl. How this is achieved is still unknown but an attractive possibility is that one of the targets of COP1-mediated degradation might directly activate PIN1 transcription. The bZIP transcription factor LONG HYPOCOTYL 5 (HY5), which is the bestcharacterized target of COP1-mediated proteolysis (Osterlund et al., 2000), would be a good candidate as several functional links between HY5 and auxin have been established (Cluis et al., 2004; Sibout et al., 2006). However, although HY5 binding sites were found in the PIN1 locus (Lee et al., 2007), genome-wide expression analyses did not detect significant changes in PIN1 expression in the hy5 mutant (Cluis et al., 2004; Zhang et al., 2011). This could still be explained by a functional redundancy between HY5 and HYH in the regulation of PIN1 (Sibout et al., 2006) but further investigations will be needed to clarify this.

Our data also demonstrate a role for COP1 in the control of PIN1 and PIN2 intracellular distribution and PM abundance in the RAM. We provide genetic evidence that COP1 regulates the decrease in both PIN1-GFP and PIN2-GFP localized at the PM, indicating a role for the ubiquitin pathway in this regulation. However, although treatment with a proteasome inhibitor in darkness clearly stabilized PIN2 association to the PM, it did not affect PIN1 localization. This suggests that the two proteins might be regulated differently, and it also points at a role of the ubiquitin pathway in the regulation of PIN2 activity by light. It has indeed 
been proposed that darkness reduces association of PIN2 to the PM by increasing degradation of the protein (Kleine-Vehn et al., 2008), similarly to what is observed during gravitropism (Abas et al., 2006; Kleine-Vehn et al., 2008). In copl mutants, we did not observe any PIN2-GFP fluorescence associated with vacuolar compartments, suggesting a possible role for COP1 in targeting PIN2 to the vacuole. In addition, we provide evidence that COP1 activity is also necessary for PIN2 degradation during the gravitropic response, a function that might be independent from the role of COP1 in photomorphogenesis. This strongly supports the idea that plants use a common module involving both COP1 and PIN2 for the regulation of root growth in response to different environmental stimuli (Kleine-Vehn et al., 2008) (our data). The existence of a permanent cytoplasmic pool of COP1 (Jang et al., 2010) raises the possibility that, in the RAM, COP1 might directly target PIN2 for degradation through ubiquitination. Such a mechanism remains to be proven but is plausible given the recent demonstration of direct ubiquitination of PIN2 during gravitropism (Leitner et al., 2012). A role for COP1 in the regulation of root growth by light, through affecting the organization of the actin cytoskeleton (Dyachok et al., 2011), has also been demonstrated. Actin is known to be essential for the intracellular trafficking of PIN proteins (Geldner et al., 2001; Boutté et al., 2006) and COP1 could affect PIN1 and PIN2 intracellular localization by modulating the activity of the actin cytoskeleton. Elucidating the mode of action of COP1 will need further experiments but our findings suggest an elegant model that explains how light regulates shoot-to-root PAT and auxin distribution over the entire plant to coordinate root and shoot development in response to light in a COP1-dependent manner (supplementary material Fig. S12).

\section{Acknowledgements}

We thank Patrick Laufs, Mark Estelle, Lars Ostergaard, Harry Klee, Takashi Ueda, Ikuko Hara-Nishimura, Arabidopsis Biological Resource Center (ABRC) and Nottingham Arabidopsis Stock Centre (NASC) for providing seeds and constructs; Isabelle Bohn-Courseau for generating the inducible CLV3 lines; and Prakash Kumar and Giorgio Morelli for valuable discussions.

\section{Funding}

M.S. was initially supported by a L'Universitá Italo Francese (UIF) Fellowship. J.X. was funded by a Netherlands Organisation for Scientific Research (NWO) Veni award; a National University of Singapore (NUS) startup fund; and Lee Hiok Kwee (LHK) donation fund. T.V. was supported by the Human Frontier Science Program Organization [HFSPO CDA 0047/2007] and the Agence National de la Recherche [AuxFate ANR-07-JCJC-0115]. This work was supported, in part, by grants from the European Research Area Networks in Systems Biology (ERASysBIO+) (iSAM); the National Key Laboratory of Crop Genetic Improvement (NKLCGI); the Italian Ministry of Education, University and Research (MIUR); the European Research Area Networks in Plant Genomics (ERA-PG) Program; and the Italian Ministry of Agriculture and Foresty (MiPAF) Agronanotech Program.

\section{Competing interests statement}

The authors declare no competing financial interests.

\section{Supplementary material}

Supplementary material available online at

http://dev.biologists.org/lookup/suppl/doi:10.1242/dev.078212/-/DC

\section{References}

Abas, L., Benjamins, R., Malenica, N., Paciorek, T., Wišniewska, J., MoulinierAnzola, J. C., Sieberer, T., Friml, J. and Luschnig, C. (2006). Intracellular trafficking and proteolysis of the Arabidopsis auxin-efflux facilitator PIN2 are involved in root gravitropism. Nat. Cell Biol. 8, 249-256.

Benková, E., Michniewicz, M., Sauer, M., Teichmann, T., Seifertová, D., Jürgens, G. and Friml, J. (2003). Local, efflux-dependent auxin gradients as a common module for plant organ formation. Cell 115, 591-602.
Bhalerao, R. P., Eklöf, J., Ljung, K., Marchant, A., Bennett, M. and Sandberg, G. (2002). Shoot-derived auxin is essential for early lateral root emergence in Arabidopsis seedlings. Plant J. 29, 325-332.

Blakeslee, J. J., Bandyopadhyay, A., Lee, O. R., Mravec, J., Titapiwatanakun, B., Sauer, M., Makam, S. N., Cheng, Y., Bouchard, R., Adamec, J. et al. (2007). Interactions among PIN-FORMED and P-glycoprotein auxin transporters in Arabidopsis. Plant Cell 19, 131-147.

Blilou, I., Xu, J., Wildwater, M., Willemsen, V., Paponov, I., Friml, J., Heidstra, R., Aida, M., Palme, K. and Scheres, B. (2005). The PIN auxin efflux facilitator network controls growth and patterning in Arabidopsis roots. Nature 433, 3944.

Boerjan, W., Cervera, M. T., Delarue, M., Beeckman, T., Dewitte, W., Bellini, C., Caboche, M., Van Onckelen, H., Van Montagu, M. and Inzé, D. (1995) Superroot, a recessive mutation in Arabidopsis, confers auxin overproduction. Plant Cell 7, 1405-1419.

Boutté, Y., Crosnier, M. T., Carraro, N., Traas, J. and Satiat-Jeunemaitre, B. (2006). The plasma membrane recycling pathway and cell polarity in plants: studies on PIN proteins. J. Cell Sci. 119, 1255-1265.

Brunoud, G., Wells, D. M., Oliva, M., Larrieu, A., Mirabet, V., Burrow, A. H. Beeckman, T., Kepinski, S., Traas, J., Bennett, M. J. et al. (2012). A novel sensor to map auxin response and distribution at high spatio-temporal resolution. Nature 482, 103-106.

Casal, J. J., Fankhauser, C., Coupland, G. and Blázquez, M. A. (2004). Signalling for developmental plasticity. Trends Plant Sci. 9, 309-314.

Ciarbelli, A. R., Ciolfi, A., Salvucci, S., Ruzza, V., Possenti, M., Carabelli, M., Fruscalzo, A., Sessa, G., Morelli, G. and Ruberti, I. (2008). The Arabidopsis homeodomain-leucine zipper II gene family: diversity and redundancy. Plant Mol. Biol. 68 , 465-478

Clough, S. J. and Bent, A. F. (1998). Floral dip: a simplified method for Agrobacterium-mediated transformation of Arabidopsis thaliana. Plant J. 16, 735-743.

Cluis, C. P., Mouchel, C. F. and Hardtke, C. S. (2004). The Arabidopsis transcription factor HY5 integrates light and hormone signaling pathways. Plant J. 38, 332-347.

Colón-Carmona, A., You, R., Haimovitch-Gal, T. and Doerner, P. (1999). Spatio-temporal analysis of mitotic activity with a labile cyclin-GUS fusion protein. Plant J. 20, 503-508.

Dello loio, R., Linhares, F. S., Scacchi, E., Casamitjana-Martinez, E., Heidstra, R., Costantino, P. and Sabatini, S. (2007). Cytokinins determine Arabidopsis root-meristem size by controlling cell differentiation. Curr. Biol. 17, 678-682.

Deveaux, Y., Peaucelle, A., Roberts, G. R., Coen, E., Simon, R., Mizukami, Y., Traas, J., Murray, J. A., Doonan, J. H. and Laufs, P. (2003). The ethanol switch: a tool for tissue-specific gene induction during plant development. Plant J. 36, 918-930.

Dyachok, J., Zhu, L., Liao, F., He, J., Huq, E. and Blancaflor, E. B. (2011). SCAR mediates light-induced root elongation in Arabidopsis through photoreceptors and proteasomes. Plant Cell 23, 3610-3626.

Ebine, K., Okatani, Y., Uemura, T., Goh, T., Shoda, K., Niihama, M., Morita, M. T., Spitzer, C., Otegui, M. S., Nakano, A. et al. (2008). A SNARE complex unique to seed plants is required for protein storage vacuole biogenesis and seed development of Arabidopsis thaliana. Plant Cell 20, 3006-3021.

Esmon, C. A., Tinsley, A. G., Ljung, K., Sandberg, G., Hearne, L. B. and Liscum, E. (2006). A gradient of auxin and auxin-dependent transcription precedes tropic growth responses. Proc. Natl. Acad. Sci. USA 103, 236-241.

Feldman, L. J. (1984). Regulation of root development. Annu. Rev. Plant Physiol. $35,223-242$

Fletcher, J. C., Brand, U., Running, M. P., Simon, R. and Meyerowitz, E. M. (1999). Signaling of cell fate decisions by CLAVATA3 in Arabidopsis shoot meristems. Science 283, 1911-1914.

Friml, J. (2010). Subcellular trafficking of PIN auxin efflux carriers in auxin transport. Eur. J. Cell Biol. 89, 231-235.

Friml, J., Vieten, A., Sauer, M., Weijers, D., Schwarz, H., Hamann, T., Offringa, R. and Jürgens, G. (2003). Efflux-dependent auxin gradients establish the apical-basal axis of Arabidopsis. Nature 426, 147-153.

Gälweiler, L., Guan, C., Müller, A., Wisman, E., Mendgen, K., Yephremov, A. and Palme, K. (1998). Regulation of polar auxin transport by AtPIN1 in Arabidopsis vascular tissue. Science 282, 2226-2230.

Geldner, N., Friml, J., Stierhof, Y. D., Jürgens, G. and Palme, K. (2001). Auxin transport inhibitors block PIN1 cycling and vesicle trafficking. Nature 413, 425428.

Geldner, N., Richter, S., Vieten, A., Marquardt, S., Torres-Ruiz, R. A., Mayer, U. and Jürgens, G. (2004). Partial loss-of-function alleles reveal a role for GNOM in auxin transport-related, post-embryonic development of Arabidopsis. Development 131, 389-400.

Grieneisen, V. A., Xu, J., Marée, A. F., Hogeweg, P. and Scheres, B. (2007). Auxin transport is sufficient to generate a maximum and gradient guiding root growth. Nature 449, 1008-1013

Henriques, R., Jang, I. C. and Chua, N. H. (2009). Regulated proteolysis in lightrelated signaling pathways. Curr. Opin. Plant Biol. 12, 49-56. 
Jensen, P. J., Hangarter, R. P. and Estelle, M. (1998). Auxin transport is required for hypocotyl elongation in light-grown but not dark-grown Arabidopsis. Plant Physiol. 116, 455-462.

Kleine-Vehn, J., Leitner, J., Zwiewka, M., Sauer, M., Abas, L., Luschnig, C. and Friml, J. (2008). Differential degradation of PIN2 auxin efflux carrier by retromer-dependent vacuolar targeting. Proc. Natl. Acad. Sci. USA 105, 17812 17817

Lau, O. S. and Deng, X. W. (2010). Plant hormone signaling lightens up: integrators of light and hormones. Curr. Opin. Plant Biol. 13, 571-577.

Laxmi, A., Pan, J., Morsy, M. and Chen, R. (2008). Light plays an essential role in intracellular distribution of auxin efflux carrier PIN2 in Arabidopsis thaliana. PLOS ONE 3, e1510

Lee, J., He, K., Stolc, V., Lee, H., Figueroa, P., Gao, Y., Tongprasit, W., Zhao, H., Lee, I. and Deng, X. W. (2007). Analysis of transcription factor HY5 genomic binding sites revealed its hierarchical role in light regulation of development. Plant Cell 19, 731-749

Leitner, J., Petrásek, J., Tomanov, K., Retzer, K., Parezová, M., Korbei, B., Bachmair, A., Zazímalová, E. and Luschnig, C. (2012). Lysine ${ }^{63}$-linked ubiquitylation of PIN2 auxin carrier protein governs hormonally controlled adaptation of Arabidopsis root growth. Proc. Natl. Acad. Sci. USA 109, 8322 8327

Li, J., Li, G., Wang, H. and Deng, X. W. (2011). Phytochrome Signaling Mechanisms. The Arabidopsis Book 9, e0148.

López-Juez, E., Dillon, E., Magyar, Z., Khan, S., Hazeldine, S., de Jager, S. M., Murray, J. A., Beemster, G. T., Bögre, L. and Shanahan, H. (2008). Distinct light-initiated gene expression and cell cycle programs in the shoot apex and cotyledons of Arabidopsis. Plant Cell 20, 947-968.

Luschnig, C., Gaxiola, R. A., Grisafi, P. and Fink, G. R. (1998). EIR1, a rootspecific protein involved in auxin transport, is required for gravitropism in Arabidopsis thaliana. Genes Dev. 12, 2175-2187.

Matesanz, S., Gianoli, E. and Valladares, F. (2010). Global change and the evolution of phenotypic plasticity in plants. Ann. N. Y. Acad. Sci. 1206, 35-55

McNellis, T. W., von Arnim, A. G., Araki, T., Komeda, Y., Miséra, S. and Deng, X. W. (1994). Genetic and molecular analysis of an allelic series of cop1 mutants suggests functional roles for the multiple protein domains. Plant Cell $\mathbf{6}$ 487-500.

Nemhauser, J. and Chory, J. (2002). Photomorphogenesis. Rockville, MD American Society of Plant Biologists.

Nicotra, A. B., Atkin, O. K., Bonser, S. P., Davidson, A. M., Finnegan, E. J., Mathesius, U., Poot, P., Purugganan, M. D., Richards, C. L., Valladares, F. et al. (2010). Plant phenotypic plasticity in a changing climate. Trends Plant Sci. $15,684-692$

Okada, K., Ueda, J., Komaki, M. K., Bell, C. J. and Shimura, Y. (1991). Requirement of the auxin polar transport system in early stages of Arabidopsis floral bud formation. Plant Cell 3, 677-684

Osterlund, M. T., Hardtke, C. S., Wei, N. and Deng, X. W. (2000). Targeted destabilization of HY5 during light-regulated development of Arabidopsis. Nature 405, 462-466.

Ottenschläger, I., Wolff, P., Wolverton, C., Bhalerao, R. P., Sandberg, G., Ishikawa, H., Evans, M. and Palme, K. (2003). Gravity-regulated differential auxin transport from columella to lateral root cap cells. Proc. Natl. Acad. Sci. USA 100, 2987-2991.

Paciorek, T., Zazímalová, E., Ruthardt, N., Petrásek, J., Stierhof, Y. D., KleineVehn, J., Morris, D. A., Emans, N., Jürgens, G., Geldner, N. et al. (2005). Auxin inhibits endocytosis and promotes its own efflux from cells. Nature $\mathbf{4 3 5}$, 1251-1256.

Parry, G., Calderon-Villalobos, L. I., Prigge, M., Peret, B., Dharmasiri, S., Itoh, H., Lechner, E., Gray, W. M., Bennett, M. and Estelle, M. (2009). Complex regulation of the TIR1/AFB family of auxin receptors. Proc. Natl. Acad. Sci. USA 106, 22540-22545.

Pfaffl, M. W. (2001). A new mathematical model for relative quantification in realtime RT-PCR. Nucleic Acids Res. 29, e45.

Quail, P. H. (2002). Phytochrome photosensory signalling networks. Nat. Rev. Mol. Cell Biol. 3, 85-93.

Reed, R. C., Brady, S. R. and Muday, G. K. (1998). Inhibition of auxin movement from the shoot into the root inhibits lateral root development in Arabidopsis. Plant Physiol. 118, 1369-1378.
Reinhardt, D., Pesce, E. R., Stieger, P., Mandel, T., Baltensperger, K., Bennett M., Traas, J., Friml, J. and Kuhlemeier, C. (2003). Regulation of phyllotaxis by polar auxin transport. Nature 426, 255-260

Rosado, A., Sohn, E. J., Drakakaki, G., Pan, S., Swidergal, A., Xiong, Y., Kang, B. H., Bressan, R. A. and Raikhel, N. V. (2010). Auxin-mediated ribosomal biogenesis regulates vacuolar trafficking in Arabidopsis. Plant Cell 22, 143-158.

Salisbury, F. J., Hall, A., Grierson, C. S. and Halliday, K. J. (2007). Phytochrome coordinates Arabidopsis shoot and root development. Plant J. 50, 429-438.

Sauer, M., Balla, J., Luschnig, C., Wisniewska, J., Reinöhl, V., Friml, J. and Benková, E. (2006). Canalization of auxin flow by Aux/IAA-ARF-dependent feedback regulation of PIN polarity. Genes Dev. 20, 2902-2911.

Scarpella, E., Francis, P. and Berleth, T. (2004). Stage-specific markers define early steps of procambium development in Arabidopsis leaves and correlate termination of vein formation with mesophyll differentiation. Development 131, 3445-3455

Shirakawa, M., Ueda, H., Shimada, T., Nishiyama, C. and Hara-Nishimura, I. (2009). Vacuolar SNAREs function in the formation of the leaf vascular network by regulating auxin distribution. Plant Cell Physiol. 50, 1319-1328.

Sibout, R., Sukumar, P., Hettiarachchi, C., Holm, M., Muday, G. K. and Hardtke, C. S. (2006). Opposite root growth phenotypes of hy5 versus hy5 hyh mutants correlate with increased constitutive auxin signaling. PLoS Genet. 2, e202.

Sieberer, T., Seifert, G. J., Hauser, M. T., Grisafi, P., Fink, G. R. and Luschnig C. (2000). Post-transcriptional control of the Arabidopsis auxin efflux carrier EIR1 requires AXR1. Curr. Biol. 10, 1595-1598.

Stepanova, A. N., Robertson-Hoyt, J., Yun, J., Benavente, L. M., Xie, D. Y., Dolezal, K., Schlereth, A., Jürgens, G. and Alonso, J. M. (2008). TAA1mediated auxin biosynthesis is essential for hormone crosstalk and plant development. Cell 133, 177-191.

Tamura, K., Shimada, T., Ono, E., Tanaka, Y., Nagatani, A., Higashi, S. I., Watanabe, M., Nishimura, M. and Hara-Nishimura, I. (2003). Why green fluorescent fusion proteins have not been observed in the vacuoles of higher plants. Plant J. 35, 545-555.

Tao, Y., Ferrer, J. L., Ljung, K., Pojer, F., Hong, F., Long, J. A., Li, L., Moreno, J. E., Bowman, M. E., Ivans, L. J. et al. (2008). Rapid synthesis of auxin via a new tryptophan-dependent pathway is required for shade avoidance in plants. Cell 133, 164-176.

Ulmasov, T., Murfett, J., Hagen, G. and Guilfoyle, T. J. (1997). Aux/IAA proteins repress expression of reporter genes containing natural and highly active synthetic auxin response elements. Plant Cell 9, 1963-1971.

Vernoux, T., Kronenberger, J., Grandjean, O., Laufs, P. and Traas, J. (2000). PIN-FORMED 1 regulates cell fate at the periphery of the shoot apical meristem. Development 127, 5157-5165.

Vieten, A., Vanneste, S., Wisniewska, J., Benková, E., Benjamins, R., Beeckman, T., Luschnig, C. and Friml, J. (2005). Functional redundancy of PIN proteins is accompanied by auxin-dependent cross-regulation of PIN expression. Development 132, 4521-4531.

Wisniewska, J., Xu, J., Seifertová, D., Brewer, P. B., Ruzicka, K., Blilou, I., Rouquié, D., Benková, E., Scheres, B. and Friml, J. (2006). Polar PIN localization directs auxin flow in plants. Science 312, 883

Xu, J. and Scheres, B. (2005). Dissection of Arabidopsis ADP-RIBOSYLATION FACTOR 1 function in epidermal cell polarity. Plant Cell 17, 525-536.

Xu, J., Hofhuis, H., Heidstra, R., Sauer, M., Friml, J. and Scheres, B. (2006). A molecular framework for plant regeneration. Science 311, 385-388.

Yi, C. and Deng, X. W. (2005). COP1-from plant photomorphogenesis to mammalian tumorigenesis. Trends Cell Biol. 15, 618-625.

Zádníková, P., Petrásek, J., Marhavy, P., Raz, V., Vandenbussche, F., Ding, Z. Schwarzerová, K., Morita, M. T., Tasaka, M., Hejátko, J. et al. (2010). Role of PIN-mediated auxin efflux in apical hook development of Arabidopsis thaliana. Development 137, 607-617

Zhang, H., He, H., Wang, X., Wang, X., Yang, X., Li, L. and Deng, X. W. (2011). Genome-wide mapping of the HY5-mediated gene networks in Arabidopsis that involve both transcriptional and post-transcriptional regulation. Plant J. 65, 346-358.

Zhao, Y., Christensen, S. K., Fankhauser, C., Cashman, J. R., Cohen, J. D., Weigel, D. and Chory, J. (2001). A role for flavin monooxygenase-like enzymes in auxin biosynthesis. Science 291, 306-309. 